second law of thermodynamics is not violated, as it would be if the diaphragm effected only an unequal distribution of energy. The great point, however, to notice is that the sifting power of the diaphragm enables us to derive work from the gas at the expense of its heat, or we obtain thereby a capacity for work without the performance of work, which is the practical result we require, and so long as we obtain this restult, we may not care so much about any inquiry whether a certain statement of a law is thereby violated or not (at least this inquiry is of secondary importance). The main point evidently is to realise how work may be derived from norinal temperature heat without a source or refrigerator. Also it cannot surely be kept too much in view that the "second law of thermodynamics" is not theoretically a necessary truth, but its truth only depends (as Prof. Maxwell showed) on our inability to grasp or handle molecules. For if molecules were of such a size that we could handle them separately, then there is no doubt that we could transfer their motions to masses (without the necessity for mixing the molecules of different masses together). The attempts to prove the second law of thermodynamics as an abstract truth independently of considering the molecular state of matter, can therefore scarcely be considered as legitimate, as it is upon the molecular state of matter that the impracticability of the effect expressed in the law depends. Also it wonld be, perhaps, difficult to give a perfectly satisfactory a priori proof that no process can be discovered for utilising normal temperature heat without permanently mixing or altering the distribution of the matter concerned, more especially when it is considered how much can be already done in the way of manipulating molecules (or sifting their velocities) and deriving their heat by means of porous diaphragms in the case of diffusion. The practicability of the result would admittedly not be contrary to the principle of the conservation of energy.

6 . One of the most important considerations, perhaps, comnected with diffusion, would appear to be that the tendency to the uniform diffusion of matter, or rather of velocity [since chemically different molecules of equal mass do not necessarily tend to become uniformly diffised], can upset the tendency to the uniform diffusion of energy, i.c., energy could not be uniformly diffused until matter (capable of diffusion) was also miformily diffused, or homogeneous. Another important consideration would appenr to be (and which, if noticed, would seem to be worthy of greater attention) that the gases of the atmosphere from the fact of their being of different molecular weights, tend forcibly to become uniformly diffused, or the danger of unequal mixture is averted, which would inevitably occur sometime or somewhere, if the gases were of the same molecular weights (or dynamically alike), and so diffusion were left to the pure contingencies of chance. I may return (by permission of the Editor) to this point at a future opportunity.

S. Tolver Preston

\section{"Underground Temperature"}

Tre: Report of the British Association Committee on Underground Temperature appearing in NATURE, vol. xvii. p. 476, gives me an opportunity of questioning the treatment of the matter and urging the rejection of any figures obtained. This opportunity I had often wished for when reading over the allusions to underground temperature which spoil text-books, but would scarcely avail myself of now were not the "report" apparently "accepted" in significant silence.

To any one familiar with the state of circumstances down in mines, who has accumulated thought on the question of the temperature of the rocks in depth, the observations noted in this report mast appear, not to say absolutely inadequate to further the inquiry, but altogether missing the point of it-that is to say, if I am right as I take it, that the purpose of the Committee is to ascertain, not mere "underground temperature" readings, but the proper tempera. ture of the rocks as due to intra-terrestrial conclitions. In all the observations conducted in mines, the temperature of the mine ventilation or a temperature almost wholly inter-dlependent is expressed by the figures obtained ; these figures no more indicate the true state of the case, anch are therefore of no more value to the geologist or the general physicist, than the tempera ture of a greenhouse would assist the meteorologist. The figures obtained in bores express the temperature of the waters stancling therein - which temperature cannot at all be assumed to be coincident with the rock temperature; these figures are de termined by a variety of factors, the true rock temperature not being 'necessarily the greater. In fact, wo can never arrive at the temperature of the rocks in depth through the media of water or air. To state this truth is to establish it.

A few remarks, however, bearing directly on the details of the observations in the report, may aid inquirers to arrive at a true state of thourht on the subject. In the first place, and as of general application, $I$ have to demand attention to the matter of the mine area, to depths exceeding the observation stations, being depleted of the waters naturally appertaining theretoand for this reason even a rock temperature ascertained over such an area would be abnormal. A thermometer bung up in a mine way will unquestionably register the temperature of the ventilation in that particular place; nobody expects any other result. Can the temperature of the ventilation be demonstrated to be inter-dependent in a great measure on the temperature of the surrounding rocks, which is itself abnormal as above submitted? Certainly not! and this fact is so obvious to those having a true acquaintance with mine ventilation, that it seems to me too absurd to elaborate proofs of it. Particular stress is laid on the point that currents of air and well-aired situations were avoided. It is hard to see any outcome of this other than still more abnormal readings. Surely observers do not imagine they can penetrate the rocks so far by cornering in mines as to leave the atmosphere wholly behind. Figures obtained in still air express the temperature of dying ventilation, or of gases of exudation, or partly of both. As to the employ. ment of a few inches of water in a hole, as supposed to secure more direct contact with the rocks, or to isolate from air ; the tempe rature of the small body of water so employed is that obtained, and it is obvious it depends on the air temperature, and (worse) the water may possibly be decomposing. These remarks apply, for the most part, alike to the ingenious method employed down Boldon Colliery as to the more simple method of Schemnitz. Then, with regard to the Boldon Colliery observations in particular, the curious in these matters will be led to speculate as to what was going on up the ten-foot hole, and whether the "stagnant" ventilation of the district was not tending towards the explosive conditions. All the circumstances previously set down, supplemented by the exudation of gases, and the sub. sidence of strata following on coal working, combine to render this one of the unhappiest conceivable situations for the research on rock temperature.

A thermometer down a mine is of no utility beyond qualifying barometric readings.

The bore observations are, I venture to think, a crude phase of the method which may lead to success ultimately. As hitherto conducted, they are open to many obvious objections, which, if stated at length, would be little more than a reiteration of the above in part.

I will venture to suggest that the next steps in advance be the permanent placing of instruments in deep bores, broken rock heing rammed over as over powder in blasting operations, so that all water and air, except such as may be fairly considered as entering into the structure of the rocks, be entirely excluded, and the application of thermo-electric apparatus devised by specialists in electric science, all constituting a special, and, for many reasons, invaluable attachment to an observatory or kindred institution.

EarIshill Colliery, Thurles

\section{Helmholtz's Vowel Theory and the Phonograph}

THE results obtained by Messrs. Jenkin and Ewing in their experiments with the phonograph, as described in NATURE, vol. xvii. p. 384 , are so different from those reached in some experi ments recently performed by Dr. Clarence J. Blake, of this city, in comection with myself, that I venture to call attention to the fact.

With the design of testing the question of change of quality in vowel toncs by increasing the rate of rotation of the phonograph cylinder, we performed a number of experiments, of which I mention a few as briefly as possible.

I. The vowels ou and $\bar{o}$ were spoken into the mouth-piece of the instrument, each four times in succession, while the cylinder was rotated at the rate of one revolution per second, as timed by the beats of a clock-pendulum. On rotating the disc so as to reproduce the vowel-soinds, these were as spoken, ou, o, each repeated four times, when the rate of rotation was one revolution per second, but on increasing the velocity to two revolutions per second, the first sounds were indistinct, while the last gave the 